\title{
ANN Synthesis Model of Single-Feed Corner-Truncated Circularly Polarized Microstrip Antenna with an Air Gap for Wideband Applications
}

\author{
Zhongbao Wang and Shaojun Fang \\ School of Information Science and Technology, Dalian Maritime University, Dalian, Liaoning 116026, China \\ Correspondence should be addressed to Shaojun Fang; fangshj@dlmu.edu.cn
}

Received 15 October 2013; Accepted 20 January 2014; Published 25 February 2014

Academic Editor: Francisco Falcone

Copyright (C) 2014 Z. Wang and S. Fang. This is an open access article distributed under the Creative Commons Attribution License, which permits unrestricted use, distribution, and reproduction in any medium, provided the original work is properly cited.

A computer-aided design model based on the artificial neural network (ANN) is proposed to directly obtain patch physical dimensions of the single-feed corner-truncated circularly polarized microstrip antenna (CPMA) with an air gap for wideband applications. To take account of the effect of the air gap, an equivalent relative permittivity is introduced and adopted to calculate the resonant frequency and $Q$-factor of square microstrip antennas for obtaining the training data sets. ANN architectures using multilayered perceptrons (MLPs) and radial basis function networks (RBFNs) are compared. Also, six learning algorithms are used to train the MLPs for comparison. It is found that MLPs trained with the Levenberg-Marquardt (LM) algorithm are better than RBFNs for the synthesis of the CPMA. An accurate model is achieved by using an MLP with three hidden layers. The model is validated by the electromagnetic simulation and measurements. It is enormously useful to antenna engineers for facilitating the design of the single-feed CPMA with an air gap.

\section{Introduction}

Wideband circularly polarized microstrip antennas (CPMAs) have been widely adopted in radio frequency identification readers [1-3], global navigation satellite systems $[4,5]$, and mobile satellite communication systems [6,7]. Generally, the single-feed and dual-feed structures are used in CPMAs. The dual-feed structure provides a wide axial ratio (AR) bandwidth, but it requires a large ground plane size for the feed network [8]. The single-feed configuration only involves slightly perturbing the patch structure at appropriate locations to excite two orthogonal modes with a $90^{\circ}$ phase shift for $\mathrm{CP}$ radiation. Some ingenious perturbation techniques have been reported to generate circularly polarized $(\mathrm{CP})$ radiation of single-feed CPMAs, such as the diagonal-feed near square, square with stubs or notches along the two opposite edges, corner-truncated squares, and squares with a diagonal slot [9]. The well-known method of producing CP radiation of a single-feed square microstrip antenna by symmetrically truncating a pair of patch corners [10] has been widely used. It has the advantages of simple structure, easy manufacture, and low cost. However, it has inherently narrow AR bandwidth of $0.5-1 \%[10,11]$. For a wider AR bandwidth, an air gap has been introduced below the radiating patch [12] or the dielectric substrate [13]. The CP performance of single-feed CPMAs with different thickness of the air gap is reported in [14], and the AR bandwidth is enhanced to $14 \%$. In addition, tuning the air gap is a simple method of changing the resonant frequency of the microstrip antenna [15], without resorting to a new antenna.

The design theory of the single-feed corner-truncated CPMA has been reported by Haneishi and Yoshida in 1981 [10]. In the design process, the resonant frequency and $Q$ factor of the square microstrip antenna must be determined by empirical formulas [16-18], electromagnetic simulation, or practical measurement. It is well known that the electromagnetic simulation is inefficient, and the practical measurement is costly. So the empirical formulas constitute one of the feasible solutions to calculate the resonant frequency and Q-factor. However, empirical formulas are mathematically 
complex and the patch physical dimensions cannot be directly calculated. Therefore, a straightforward computeraided design (CAD) model is required to facilitate the antenna design. In addition, a modification of the empirical formulas is necessary in order to take account of the effect of the air gap.

Recently, CAD models based on artificial neural networks (ANNs) have been presented for the analysis and the synthesis of microstrip antennas in various forms such as rectangular, square, and circular patches [19-23]. The analysis model is used to determine the resonant frequency for a given dielectric material and patch structure. The synthesis model can be built with ANN inverse modeling method [24, 25] to compute patch physical dimensions for the required design specifications. An overview of the development of the analysis and the synthesis of microstrip antennas with the ANN has been reported in [26]. There are only synthesis models for linearly polarized microstrip antennas. Up to date, an ANN synthesis model for a single-feed CPMA is published by Wang et al. [27]. However, the model is only for narrow-band CPMAs. And the inputs of the model are $H / \lambda_{g}$ and $\tau_{d}$ rather than $H$ and $\tan \delta$. A transformation is needed before the use of the model, which is not too easy to use.

In this paper, a user-friendly synthesis model of the single-feed corner-truncated CPMA with an air gap is proposed for wideband applications. First, an equivalent relative permittivity $\varepsilon_{\mathrm{eq}}$ is introduced to take account of the effect of the air gap. Using the $\varepsilon_{\text {eq }}$, the square microstrip antenna with an air gap (SMAAG) is analyzed to obtain the values of the resonant frequency and $Q$-factor. Then, the size of the truncated corners and the value of the $\mathrm{CP}$ operation frequency with the best $\mathrm{AR}$ are calculated to obtain the training and test data sets. Next, MLPs and RBFNs are used to construct the synthesis model for finding a proper ANN architecture. And an accurate model is achieved by using an MLP with three hidden layers. At last, the results of the synthesis model are compared with the HFSS simulation [28] and measurements. A good agreement among the results of the synthesis model, HFSS simulation, and measurements is obtained.

\section{Theoretical Formulations}

Figure 1 shows the configuration of the single-feed cornertruncated CPMA with an air gap. A probe-feed square patch with a dimension of $L \times L$ is printed on a dielectric substrate with a thickness of $H_{d}$, relative permittivity of $\varepsilon_{r}$, and loss $\operatorname{tangent}$ of $\tan \delta$. There is an air gap between the dielectric substrate and the ground plane. The height of the air gap is $H_{a}$. A pair of patch corners shown in Figure 1(b) is symmetrically truncated to produce $\mathrm{CP}$ radiation.

2.1. Equivalent Relative Permittivity. Here, we model the square microstrip antenna with an air gap by an equivalent single-layer structure of the total height $H_{t}=H_{a}+H_{d}$ with an equivalent relative permittivity $\varepsilon_{\mathrm{eq}}$ :

$$
\varepsilon_{\mathrm{eq}}=\frac{2 \varepsilon_{\mathrm{eff}}-1+A}{1+A}
$$

$$
A=\left(1+\frac{12 H_{t}}{L}\right)^{-1 / 2}
$$

where $\varepsilon_{\text {eff }}$ is effective relative permittivity [29].

2.2. Resonant Frequency of SMAAG. Up to now, formulas based on transmission-line, cavity, and magnetic-wall models have been presented to determine the resonant frequency of the square microstrip antenna [30]. The measurement performed in $[18,30]$ has demonstrated that the formula based on the transmission-line model (TLM) is accurate for the substrate thickness in the range of $H \leq 0.1 \lambda_{g}$. When a square microstrip antenna with an air gap is operating at its fundamental mode (i.e., $\mathrm{TM}_{10}$ mode), the formula based on the TLM to calculate the resonant frequency is modified as

$$
f_{r}=\frac{c}{2 L_{e} \sqrt{\varepsilon_{\mathrm{eq}}}},
$$

where $c$ is the velocity of electromagnetic waves in free space and $L_{e}$ is the effective length of the square patch. The effective length $L_{e}$ is obtained by adding the Hammerstad fringing length [31] to each end of the patch. It should be noted that the usage limitation of the formula (2) for the SMAAG is given by

$$
H_{t} \leq 0.1 \lambda_{\text {eq }}=0.1 \frac{\lambda_{0}}{\sqrt{\varepsilon_{\mathrm{eq}}}} .
$$

2.3. Q-Factor of SMAAG. The total quality factor $Q_{t}$ of the SMAAG can be expressed in terms of the $Q$-factors associated with the dielectric loss $\left(Q_{d}\right)$, conductor loss $\left(Q_{c}\right)$, radiation into space $\left(Q_{\mathrm{sp}}\right)$, and surface-wave loss $\left(Q_{\mathrm{sw}}\right)$, through the relation [18]:

$$
Q_{t}=\left(\frac{1}{Q_{d}}+\frac{1}{Q_{c}}+\frac{1}{Q_{s p}}+\frac{1}{Q_{s w}}\right)^{-1}
$$

The formulas for $Q_{d}$ and $Q_{c}$ are well known [16]. $Q_{\text {sp }}$ and $Q_{s w}$ can be computed from the expressions of Jackson et al. [18].

2.4. CP Operation Conditions. Based on the theory presented by Haneishi and Yoshida [10], the CP operation conditions for the single-feed corner-truncated CPMA with an air gap are rewritten as follows:

$$
\begin{gathered}
L_{t}=\frac{L}{\sqrt{2 Q_{t}}}, \\
f_{0}=f_{r}\left(1+\frac{1}{4 Q_{t}}\right),
\end{gathered}
$$

where $L_{t}$ is the size of the truncated corners and $f_{0}$ is the CP operation frequency with the best AR.

Noting that when the required CP operation frequency $f_{0}$ is given and the parameters of the dielectric substrate and the air gap $\left(\varepsilon_{r}, \tan \delta, H_{d}\right.$, and $\left.H_{a}\right)$ are supplied, the physical dimensions of the corner-truncated square patch $(L$ and $L_{t}$ ) cannot be directly obtained from (2)-(6). To solve this problem, ANN inverse modeling method $[24,25]$ is used to construct the nonlinear relation between $\left[f_{0}, \varepsilon_{r}, \tan \delta, H_{d}\right.$, $\left.H_{a}\right]$ and $\left[L, L_{t}\right]$. 


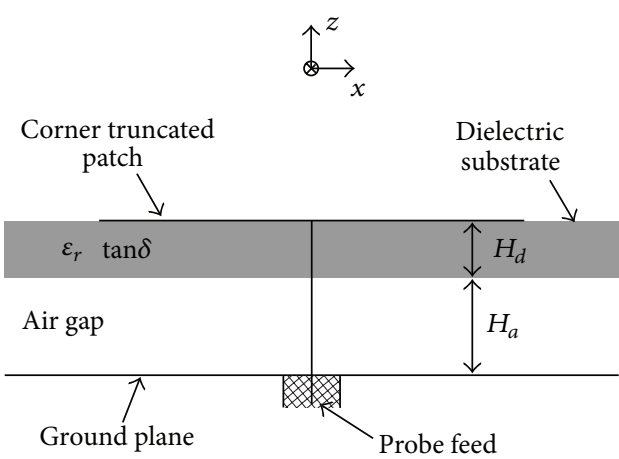

(a)

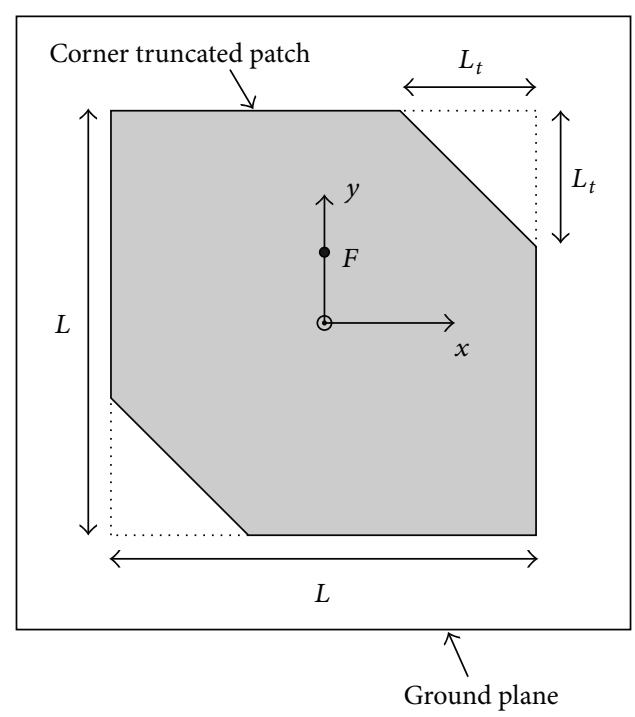

(b)

FIGURE 1: Geometry of single-feed corner-truncated CPMA with an air gap: (a) side view, (b) top view.

\section{ANN Synthesis Model}

ANNs were being developed for many years. Recently, the ANN has gained attention as a fast and flexible vehicle for microwave modeling and optimization [32]. Two types of ANNs (i.e., MLPs and RBFNs) are universal approximators. They have been widely used to construct the analysis and synthesis models for linearly polarized microsrtip antennas [19-23]. An MLP consists of three types of layers: an input layer, an output layer, and one or more hidden layers. The topology of the RBFN is similar to that of the MLP with one hidden layer. The differences lie in the characteristics of the hidden neurons, and the success of MLPs for a particular problem depends on the adequacy of the learning algorithm. To obtain a high-precision synthesis model, backpropagation with momentum (BPM), resilient backpropagation (RBP), conjugate gradient with Fletcher-Reeves (CGF), scaled conjugate gradient (SCG), Broydon-Fletcher-Goldfarb-Shanno (BFGS), and Levenberg-Marquardt (LM) algorithms [32] will be used in this study to train the MLPs. And the MLP-based model will be compared with the RBFN-based model.

The aim of this paper is to develop an accurate synthesis model for the single-feed corner-truncated CPMA with an air gap. Figure 2 gives an ANN synthesis model. The model can be used to calculate the physical dimensions of the corner-truncated square patch ( $L$ and $L_{t}$ ) for the required $\mathrm{CP}$ operation frequency $f_{0}$ with the given parameters of the dielectric substrate and the air gap $\left(\varepsilon_{r}, \tan \delta, H_{d}\right.$, and $\left.H_{a}\right)$.

The accuracy of the ANN model depends on the data sets used during training. To obtain the training and test data sets, the formulas (2)-(6) are used to generate data for $0.2 \mathrm{~mm} \leq H_{a} \leq 30.0 \mathrm{~mm}, 0.2 \mathrm{~mm} \leq H_{d} \leq 5.0 \mathrm{~mm}, 1 \leq \varepsilon_{r} \leq$ $12,10^{-4} \leq \tan \delta \leq 10^{-1.5}$, and $5 \mathrm{~mm} \leq L \leq 150 \mathrm{~mm}$. The generated data are then arranged into the input and output matrices. A five-row matrix containing the values of $H_{a}, H_{d}$, $\varepsilon_{r}, \tan \delta$, and $f_{0}$ is used as the input matrix; a two-row matrix

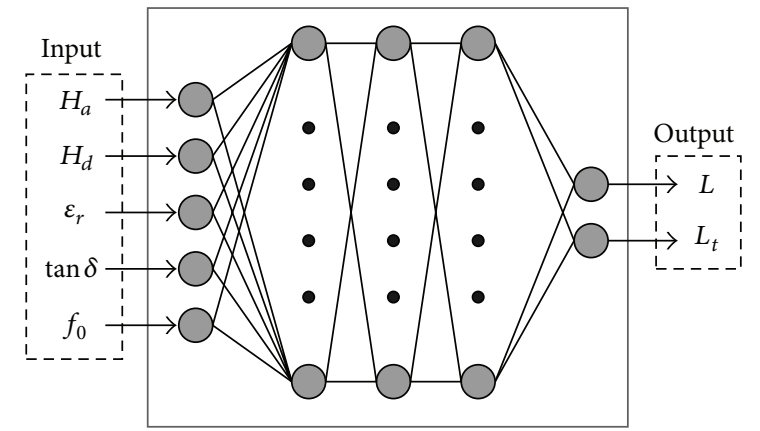

FIGURE 2: ANN synthesis model for the single-feed cornertruncated CPMA with an air gap.

containing the corresponding values of $L$ and $L_{t}$ is used as the output matrix. Due to the usage limitation of $H_{t} / \lambda_{\text {eq }} \leq 0.1$ for empirical formulas presented in Section 2, the corresponding data for $H_{t} / \lambda_{\text {eq }}>0.1$ is removed from the input and output matrixes. To facilitate an easier training process, the elements of each row of the input and output matrixes are scaled with respect to the row minimum and maximum values (e.g., 0.2 and 30 for the row, including the values of $H_{a}$ ) to transform their range to $[-1,1]$ before training. In this study, out of the 10,000 data sets generated, 7,000 are used for training and the rest are used to test the trained ANN.

For the ANN to be an accurate synthesis model of the microstip antenna, a suitable number of hidden neurons are needed. To find a proper hidden layer configuration for the synthesis model of the single-feed corner-truncated CPMA with an air gap, many experiments are carried out in this study. After many trials, it is found that the target of high accuracy is achieved by using an MLP with three hidden layers. The suitable hidden layer configuration for the synthesis model is $15 \times 20 \times 4$. It means that the numbers 
TABLE 1: Comparison of MLP-based synthesis model trained with different training algorithms.

\begin{tabular}{|c|c|c|c|c|c|c|c|c|}
\hline \multicolumn{3}{|c|}{ Training algorithm } & BPM & RBP & CGF & SCG & BFGS & LM \\
\hline \multicolumn{3}{|c|}{ Training time (seconds) } & 77 & 80 & 193 & 158 & 292 & 912 \\
\hline \multirow{4}{*}{ MRE (\%) } & \multirow{2}{*}{$L$} & Training & 321 & 79.7 & 95.6 & 92.4 & 14.1 & 1.56 \\
\hline & & Test & 214 & 49.4 & 67.8 & 72.6 & 34.6 & 1.55 \\
\hline & \multirow{2}{*}{$L_{t}$} & Training & 163 & 279 & 163 & 70.2 & 41.2 & 2.05 \\
\hline & & Test & 185 & 102 & 46.3 & 78.8 & 42.3 & 2.19 \\
\hline \multirow{4}{*}{$\operatorname{ARE}(\% o)$} & \multirow{2}{*}{$L$} & Training & 155 & 23.2 & 19.0 & 12.3 & 2.79 & 0.206 \\
\hline & & Test & 156 & 23.6 & 18.3 & 12.3 & 2.82 & 0.224 \\
\hline & \multirow{2}{*}{$L_{t}$} & Training & 123 & 27.2 & 22.6 & 16.5 & 4.49 & 0.367 \\
\hline & & Test & 121 & 26.7 & 21.8 & 16.1 & 4.60 & 0.403 \\
\hline \multirow{4}{*}{ MSE (mm) } & \multirow{2}{*}{$L$} & Training & 285 & 7.96 & 4.67 & 1.76 & $9.85 \times 10^{-2}$ & $5.96 \times 10^{-4}$ \\
\hline & & Test & 294 & 8.59 & 4.78 & 1.88 & $1.09 \times 10^{-1}$ & $8.13 \times 10^{-4}$ \\
\hline & \multirow{2}{*}{$L_{t}$} & Training & 5.97 & $2.38 \times 10^{-1}$ & $1.66 \times 10^{-1}$ & $8.64 \times 10^{-2}$ & $6.40 \times 10^{-3}$ & $4.23 \times 10^{-5}$ \\
\hline & & Test & 5.61 & $2.54 \times 10^{-1}$ & $1.57 \times 10^{-1}$ & $8.74 \times 10^{-2}$ & $7.16 \times 10^{-3}$ & $6.46 \times 10^{-5}$ \\
\hline
\end{tabular}

TABLE 2: Comparison of MLP-based synthesis model with different hidden layer configurations.

\begin{tabular}{|c|c|c|c|c|c|c|c|c|}
\hline \multicolumn{3}{|c|}{ Configuration of hidden layers } & \multicolumn{3}{|c|}{ Two hidden layers } & \multicolumn{3}{|c|}{ Three hidden layers } \\
\hline & & & $15 \times 14$ & $15 \times 20$ & $15 \times 26$ & $15 \times 20 \times 2$ & $15 \times 20 \times 4$ & $15 \times 20 \times 6$ \\
\hline \multicolumn{3}{|c|}{ Training time (seconds) } & 606 & 871 & 1146 & 827 & 912 & 1074 \\
\hline \multirow{4}{*}{ MRE (\%) } & \multirow{2}{*}{$L$} & Training & 2.56 & 2.25 & 5.16 & 2.04 & 1.56 & 1.19 \\
\hline & & Test & 3.37 & 2.30 & 5.66 & 1.41 & 1.55 & 1.35 \\
\hline & \multirow{2}{*}{$L_{t}$} & Training & 7.82 & 4.12 & 3.88 & 2.87 & 2.05 & 1.29 \\
\hline & & Test & 10.3 & 5.14 & 13.3 & 2.03 & 2.19 & 3.48 \\
\hline \multirow{4}{*}{$\operatorname{ARE}(\%)$} & \multirow{2}{*}{$L$} & Training & 0.491 & 0.473 & 0.754 & 0.288 & 0.206 & 0.190 \\
\hline & & Test & 0.519 & 0.486 & 0.820 & 0.298 & 0.224 & 0.221 \\
\hline & \multirow{2}{*}{$L_{t}$} & Training & 0.928 & 0.846 & 0.140 & 0.505 & 0.367 & 0.387 \\
\hline & & Test & 0.968 & 0.871 & 0.141 & 0.540 & 0.403 & 0.415 \\
\hline \multirow{4}{*}{$\operatorname{MSE}(\mathrm{mm})$} & \multirow{2}{*}{$L$} & Training & $3.60 \times 10^{-3}$ & $3.43 \times 10^{-3}$ & $7.85 \times 10^{-3}$ & $1.15 \times 10^{-3}$ & $5.96 \times 10^{-4}$ & $5.17 \times 10^{-4}$ \\
\hline & & Test & $4.77 \times 10^{-3}$ & $4.17 \times 10^{-3}$ & $9.94 \times 10^{-3}$ & $1.31 \times 10^{-3}$ & $8.13 \times 10^{-4}$ & $8.09 \times 10^{-4}$ \\
\hline & \multirow{2}{*}{$L_{t}$} & Training & $2.95 \times 10^{-3}$ & $2.46 \times 10^{-4}$ & $6.39 \times 10^{-4}$ & $8.90 \times 10^{-5}$ & $4.23 \times 10^{-5}$ & $5.07 \times 10^{-5}$ \\
\hline & & Test & $3.38 \times 10^{-3}$ & $2.62 \times 10^{-4}$ & $7.71 \times 10^{-4}$ & $1.11 \times 10^{-4}$ & $6.46 \times 10^{-5}$ & $6.27 \times 10^{-5}$ \\
\hline
\end{tabular}

TABLE 3: Comparison of the synthesis model constructed with MLPs and RBFNs.

\begin{tabular}{|c|c|c|c|c|c|c|c|c|}
\hline & Jetw & & MLP & & & RBFNs & & \\
\hline Hic & yer & ion & $15 \times 20 \times 4$ & 100 & 200 & 300 & 400 & 500 \\
\hline & tim & & 912 & 208 & 452 & 714 & 1032 & 1378 \\
\hline & $I$ & Training & 1.56 & 356 & 347 & 214 & 132 & 61.8 \\
\hline MRE (\%) & $\mathrm{L}$ & Test & 1.55 & 225 & 191 & 115 & 528 & 1573 \\
\hline & $L$ & Training & 2.05 & 260 & 196 & 59.5 & 65.6 & 29.3 \\
\hline & & Test & 2.19 & 148 & 118 & 55.9 & 158 & 471 \\
\hline & $I$ & Training & 0.206 & 71.7 & 39.9 & 29.3 & 21.5 & 16.4 \\
\hline$A R$ & $L$ & Test & 0.224 & 70.3 & 39.8 & 31.0 & 26.6 & 28.1 \\
\hline & $L$ & Training & 0.367 & 44.6 & 23.1 & 15.9 & 11.9 & 9.0 \\
\hline & $\mathrm{L}_{t}$ & Test & 0.403 & 42.9 & 23.3 & 17.0 & 14.2 & 13.6 \\
\hline & $I$ & Training & $5.96 \times 10^{-4}$ & 51.4 & 16.2 & 9.15 & 5.32 & 3.24 \\
\hline $\operatorname{MSE}(\mathrm{mm})$ & $L$ & Test & $8.13 \times 10^{-4}$ & 51.9 & 17.5 & 11.5 & 8.81 & 17.1 \\
\hline & $I$ & Training & $4.23 \times 10^{-5}$ & 0.738 & 0.196 & 0.095 & 0.055 & 0.033 \\
\hline & $L_{t}$ & Test & $6.46 \times 10^{-5}$ & 0.731 & 0.224 & 0.130 & 0.092 & 0.124 \\
\hline
\end{tabular}


TABLE 4: Comparisons of results of MLP-based synthesis model and HFSS simulation.

\begin{tabular}{|c|c|c|c|c|c|c|c|c|c|}
\hline \multirow{2}{*}{$\begin{array}{l}\text { Air gaps } \\
H_{a}(\mathrm{~mm})\end{array}$} & \multicolumn{3}{|c|}{ Substrate materials } & \multirow{2}{*}{$\begin{array}{c}\text { Required frequency } \\
f_{0}(\mathrm{GHz})\end{array}$} & \multicolumn{2}{|c|}{ Outputs of synthesis model } & \multicolumn{2}{|c|}{ HFSS simulation } & \multirow{2}{*}{$\left|f_{0}-f_{s}\right| / f_{0}(\%)$} \\
\hline & $H_{d}(\mathrm{~mm})$ & $\varepsilon_{r}$ & $\tan \delta$ & & $L(\mathrm{~mm})$ & $L_{t}(\mathrm{~mm})$ & $f_{s}(\mathrm{GHz})$ & $\mathrm{AR}_{s}(\mathrm{~dB})$ & \\
\hline \multirow{3}{*}{0.25} & \multirow{3}{*}{3.50} & \multirow{3}{*}{10.2} & \multirow{3}{*}{0.0023} & 1.000 & 54.70 & 4.25 & 1.019 & 0.90 & 1.90 \\
\hline & & & & 2.000 & 25.69 & 2.73 & 2.008 & 1.07 & 0.40 \\
\hline & & & & 3.000 & 16.05 & 2.13 & 2.957 & 0.53 & 1.43 \\
\hline \multirow{5}{*}{0.50} & \multirow{5}{*}{2.50} & \multirow{5}{*}{4.50} & \multirow{5}{*}{0.0020} & 1.000 & 84.09 & 7.26 & 1.010 & 1.81 & 1.00 \\
\hline & & & & 2.000 & 40.48 & 4.77 & 2.014 & 1.35 & 0.70 \\
\hline & & & & 3.000 & 26.03 & 3.77 & 3.008 & 0.69 & 0.27 \\
\hline & & & & 4.000 & 18.85 & 3.18 & 3.980 & 0.30 & 0.50 \\
\hline & & & & 5.000 & 14.63 & 2.79 & 4.922 & 1.50 & 1.56 \\
\hline
\end{tabular}

TABLE 5: Comparisons of results of MLP-based synthesis model, HFSS simulation, and measurements. $\left(H_{a}=2.5 \mathrm{~mm}, H_{d}=1.5 \mathrm{~mm}, \varepsilon_{r}=2.61\right.$, and $\tan \delta=0.003)$.

\begin{tabular}{lcccccccc}
\hline $\begin{array}{l}\text { Required frequency } \\
f_{0}(\mathrm{GHz})\end{array}$ & \multicolumn{2}{c}{ Outputs of synthesis model } & \multicolumn{2}{c}{ Simulated } & \multicolumn{2}{c}{ Measured } & $\left|f_{0}-f_{s}\right| / f_{0}(\%)$ & $\left|f_{0}-f_{m}\right| / f_{0}(\%)$ \\
\hline 1.575 & $L(\mathrm{~mm})$ & $L_{t}(\mathrm{~mm})$ & $f_{s}(\mathrm{GHz})$ & $\mathrm{AR}_{s}(\mathrm{~dB})$ & $f_{m}(\mathrm{GHz})$ & $\mathrm{AR}_{m}(\mathrm{~dB})$ & 1.21 \\
2.445 & 76.59 & 9.97 & 1.560 & 0.05 & 1.594 & 1.47 & 0.95 & 1.76 \\
3.000 & 47.77 & 7.58 & 2.402 & 0.82 & 2.487 & 0.54 & 1.72 \\
\hline
\end{tabular}

of neurons are 15, 20, and 4 for the first, second, and third hidden layers, respectively. The activation function for the hidden layers is the tangent sigmoid function, and that in the input and output layers is the linear function. Initial weights of the ANN are set up randomly. The mean square error (MSE) between the target and the output of the ANN is used to adapt the weights. The maximum allowable number of training epochs was 1000.

\section{Results and Discussion}

ANNs have been successfully adopted for the synthesis of the single-feed corner-truncated CPMA with an air gap. To obtain an accurate synthesis model, MLPs are trained with the BPM, RBP, CGF, SCG, BFGS, and LM algorithms [32] and different hidden layer configurations for MLPs and RBFNs are investigated. The training times on a computer with a 3.3$\mathrm{GHz}$ Intel Core i3-2120 CPU are recorded for comparison, and the quality of each model is evaluated with the maximal relative error (MRE), average relative error (ARE) [27], and MSE.

Table 1 gives the comparison of the MLP-based model trained with different training algorithms. It is seen that although the training time of the LM algorithm is more than that of other algorithms, the best performance of the MLP-based model is obtained by using the LM algorithm. It is also seen from Table 1 that, for the MLP-based model trained with the LM algorithm, the MRE is less than $2.2 \%$, the ARE is less than $0.5 \%$, and MSE is less than $8.2 \times$ $10^{-4} \mathrm{~mm}$. These error values obviously show that the MLP trained with the LM algorithm can be used for accurately computing patch physical dimensions of the single-feed corner-truncated CPMA with an air gap.
Table 2 gives the comparison of the MLP-based model with different hidden layer configurations. It is observed that an MLP with three hidden layers is more accurate than an MLP with two hidden layers, and the synthesis model with a suitable hidden layer configuration $(15 \times 20 \times 4)$ has higher accuracy and training efficiency. Furthermore, more hidden neurons require more training time when the number of hidden layers is constant.

Table 3 gives the comparison of the synthesis model constructed with the MLPs and RBFNs. It is seen that the performance of the RBFNs is very bad compared with the MLPs, and the number of hidden neurons for the RBFNs is much more than that for the MLPs. Thus, RBFNs are unsuitable for constructing the synthesis model of the CPMA with an air gap.

To validate the MLP-based model, the results obtained from the synthesis model are compared with the HFSS simulation in Table 4 . The patch dimensions $\left(L\right.$ and $\left.L_{t}\right)$ of the single-feed corner-truncated CPMA with an air gap for the required operation frequency $f_{0}$ with the given parameters of the dielectric substrate and the air gap $\left(\varepsilon_{r}\right.$, $\tan \delta, H_{d}$, and $H_{a}$ ) are obtained from the MLP-based model, as given in the table. Then, the CPMAs with $L, L_{t}, \varepsilon_{r}, \tan \delta$, $H_{d}$, and $H_{a}$ are analyzed by HFSS simulation to obtain the $\mathrm{CP}$ operation frequency $\left(f_{s}\right)$ with the best axial ratio $\left(\mathrm{AR}_{s}\right)$. It is observed from Table 4 that AR of all antennas is less than $1.85 \mathrm{~dB}$. This means that CPMAs can be synthesized by using the proposed MLP-based model. Furthermore, there are some discrepancies between $f_{0}$ and $f_{s}$, which are likely due to the inaccuracies from the empirical formulas presented in Section 2, ANN calculation, and HFSS simulation. However, the maximal discrepancy is $1.9 \%$, and the discrepancies can be corrected by slightly adjusting $L$. Therefore, the MLP-based model can be used to design the single-feed corner-truncated CPMA with an air gap. 
In this study, three CPMAs are fabricated on RF laminates $\left(\varepsilon_{r}=2.61, \tan \delta=0.003\right.$, and $\left.H_{d}=1.5 \mathrm{~mm}\right)$ suspended above the ground plane with an air-gap height of $H_{a}=2.5 \mathrm{~mm}$. The far-field performances of these prototypes are obtained by using the SATIMO measurement system. We also obtain the far-field performances by the HFSS simulation. In Table 5, the results obtained from the MLP-based synthesis model are compared with the HFSS simulation as well as measurements. In the table, $f_{m}$ is the measured CP operation frequency with the best axial ratio $\left(\mathrm{AR}_{m}\right)$ for patch dimensions $L$ and $L_{t}$ obtained from the MLP-based synthesis model, while $f_{s}$ and $\mathrm{AR}_{s}$ represent the CP operation frequency and the axial ratio obtained by the HFSS simulation. It is observed from Table 5 that the measured AR for the antenna prototypes is less than $1.5 \mathrm{~dB}$ and the deviation for the measured CP operation frequency is also less than $1.9 \%$. There is a good agreement among the results of the MLP-based synthesis model, HFSS simulation, and measurements.

\section{Conclusion}

An ANN synthesis model for the single-feed cornertruncated CPMA with an air gap has been presented and investigated experimentally. For the MLP-based synthesis model, the MRE is less than $2.2 \%$ and the ARE is less than $0.5 \%$. These error values obviously show that the proposed model can be used to accurately compute the patch physical dimensions ( $L$ and $L_{t}$ ) simply, rather than by the iteration technique of applying the empirical formulas or electromagnetic simulation. It is exceedingly helpful to antenna engineers for designing the single-feed cornertruncated CPMA with an air gap.

\section{Conflict of Interests}

The authors declare that there is no conflict of interests regarding the publication of this paper.

\section{Acknowledgments}

This work was supported jointly by the National Natural Science Foundation of China (nos. 61071044 and 61231006), the Scientific Research Project of the Department of Education of Liaoning Province (no. L2013196), the Fundamental Research Funds for the Central Universities (nos. 3132013053 and 3132013307), and the National Key Technologies R\&D Program of China (no. 2012BAH36B01).

\section{References}

[1] H. L. Chung, X. M. Qing, and Z. N. Chen, "A broadband circularly polarized stacked probe-fed patch antenna for UHF RFID applications," International Journal of Antennas and Propagation, vol. 2007, Article ID 76793, 8 pages, 2007.

[2] Z. N. Chen, X. M. Qing, and H. L. Chung, "A universal UHF RFID reader antenna," IEEE Transactions on Microwave Theory and Techniques, vol. 57, no. 5, pp. 1275-1282, 2009.

[3] Z. B. Wang, S. J. Fang, S. Q. Fu, and S. L. Jia, "Single-fed broadband circularly polarized stacked patch antenna with horizontally meandered strip for universal UHF RFID applications," IEEE Transactions on Microwave Theory and Techniques, vol. 59, no. 4, pp. 1066-1073, 2011.

[4] Z. B. Wang, S. J. Fang, S. Q. Fu, and S. W. Lü, "Dual-band probe-fed stacked patch antenna for GNSS applications," IEEE Antennas and Wireless Propagation Letters, vol. 8, pp. 100-103, 2009.

[5] G. Liu, L. Xu, and Y. Wang, "Modified dual-band stacked circularly polarized microstrip antenna," International Journal of Antennas and Propagation, vol. 2013, Article ID 382958, 5 pages, 2013.

[6] Z. B. Wang, S. J. Fang, S. Q. Fu, and S. L. Jia, "An inmarsat BGAN terminal patch antenna array with unequal input impedance elements and conductor-backed ACPW series-feed network," IEEE Transactions on Antennas and Propagation, vol. 60, no. 3, pp. 1642-1647, 2012.

[7] A. X. Chen, C. Yang, Z. Z. Chen, Y. J. Zhang, and Y. Y. He, "Design of multilevel sequential rotation feeding networks used for circularly polarized microstrip antenna arrays," International Journal of Antennas and Propagation, vol. 2012, Article ID 304816, 10 pages, 2012.

[8] Y.-X. Guo, K.-W. Khoo, and L. C. Ong, "Wideband circularly polarized patch antenna using broadband baluns," IEEE Transactions on Antennas and Propagation, vol. 56, no. 2, pp. 319-326, 2008.

[9] G. Kumar and K. P. Ray, Broadband Microstrip Antennas, Artech House, Norwood, Mass, USA, 2002.

[10] M. Haneishi and S. Yoshida, "A design method of circularly polarized rectangular microstrip antenna by one-feed point," Electronics \& communications in Japan, vol. 64, no. 4, pp. 46$54,1981$.

[11] P. C. Sharma and K. C. Gupta, "Analysis and optimized design of single feed circularly polarized microstrip antennas," IEEE Transactions on Antennas and Propagation, vol. 31, no. 6, pp. 949-955, 1983.

[12] F.-S. Chang, K.-L. Wong, and T.-W. Chiou, "Low-cost broadband circularly polarized patch antenna," IEEE Transactions on Antennas and Propagation, vol. 51, no. 10, pp. 3006-3009, 2003.

[13] S. M. Kim and W. G. Yang, "Single feed wideband circular polarised patch antenna," Electronics Letters, vol. 43, no. 13, pp. 703-704, 2007.

[14] S. L. S. Yang, K. F. Lee, A. A. Kishk, and K. M. Luk, "Design and study of wideband single feed circularly polarized microstrip antennas," Progress in Electromagnetics Research, vol. 80, pp. 4561,2008

[15] K.-F. Lee, K. Y. Ho, and J. S. Dahele, "Circular-disk microstrip antenna with an air gap," IEEE Transactions on Antennas and Propagation, vol. AP-32, no. 8, pp. 880-884, 1984.

[16] J. R. James, P. S. Hall, and C. Wood, Microstrip Antenna-Theory and Design, Peter Peregrinus, London, UK, 1981.

[17] I. J. Bahl and P. Bhartia, Microstrip Antennas, Artech House, Norwood, Mass, USA, 1982.

[18] D. R. Jackson, S. A. Long, J. T. Williams, and V. B. Davis, "Computer-aided design of rectangular microstrip antennas," in Advances in Microstrip and Printed Antennas, K. F. Lee and W. Chen, Eds., chapter 5, John Wiley \& Sons, New York, NY, USA, 1997.

[19] R. K. Mishra and A. Patnaik, "Neural network-based CAD model for the design of square-patch antennas," IEEE Transactions on Antennas and Propagation, vol. 46, no. 12, pp. 1890-1891, 1998. 
[20] K. Güney, Ş. Sağiroğlu, and M. Erler, “Comparison of neural networks for resonant frequency computation of electrically thin and thick rectangular microstrip antennas," Journal of Electromagnetic Waves and Applications, vol. 15, no. 8, pp. 1121$1145,2001$.

[21] C. Yildiz, S. Gultekin, K. Guney, and S. Sagiroglu, "Neural models for the resonant frequency of electrically thin and thick circular microstrip antennas and the characteristic parameters of asymmetric coplanar waveguides backed with a conductor," AEU - International Journal of Electronics and Communications, vol. 56, no. 6, pp. 396-406, 2002.

[22] N. Türker, F. Günes, and T. Yildirim, "Artificial neural design of microstrip antennas," Turkish Journal of Electrical Engineering and Computer Sciences, vol. 14, no. 3, pp. 445-453, 2006.

[23] K. Güney and N. Sarikaya, "A hybrid method based on combining artificial neural network and fuzzy inference system for simultaneous computation of resonant frequencies of rectangular, circular, and triangular microstrip antennas," IEEE Transactions on Antennas and Propagation, vol. 55, no. 3, pp. 659-668, 2007.

[24] F. Güneș and N. Türker, "Artificial neural networks in their simplest forms for analysis and synthesis of $\mathrm{RF} /$ microwave planar transmission lines," International Journal of $R F$ and Microwave Computer-Aided Engineering, vol. 15, no. 6, pp. 587599, 2005.

[25] H. Kabir, Y. Wang, M. Yu, and Q.-J. Zhang, "Neural network inverse modeling and applications to microwave filter design," IEEE Transactions on Microwave Theory and Techniques, vol. 56, no. 4, pp. 867-879, 2008.

[26] V. V. Thakare and P. Singhal, "Microstrip antenna design using artificial neural networks," International Journal of $R F$ and Microwave Computer-Aided Engineering, vol. 20, no. 1, pp. 7686, 2010.

[27] Z. B. Wang, S. J. Fang, Q. Wang, and H. M. Liu, "An ANN-based synthesis model for the single-feed circularly-polarized square microstrip antenna with truncated corners," IEEE Transactions on Antennas and Propagation, vol. 60, no. 12, pp. 5989-5992, 2012.

[28] User's Guide-High Frequency Structure Simulator, Ansoft Corporation, Pittsburgh, Pa, USA, 2005.

[29] S.-S. Zhong, G. Liu, and G. Qasim, "Closed form expressions for resonant frequency of rectangular patch antennas with multidielectric layers," IEEE Transactions on Antennas and Propagation, vol. 42, no. 9, pp. 1360-1363, 1994.

[30] M. Kara, "The resonant frequency of rectangular microstrip antenna elements with various substrate thicknesses," Microwave and Optical Technology Letters, vol. 11, no. 2, pp. 55-59, 1996.

[31] E. O. Hammerstad, "Equations for microstrip circuits design," in Proceedings of 5th European Microwave Conference, pp. 268272, Hamburg, Germany, 1975.

[32] Q. J. Zhang and K. C. Gupta, Neural Networks for RF and Microwave Design, Artech House, Norwood, Mass, USA, 2000. 

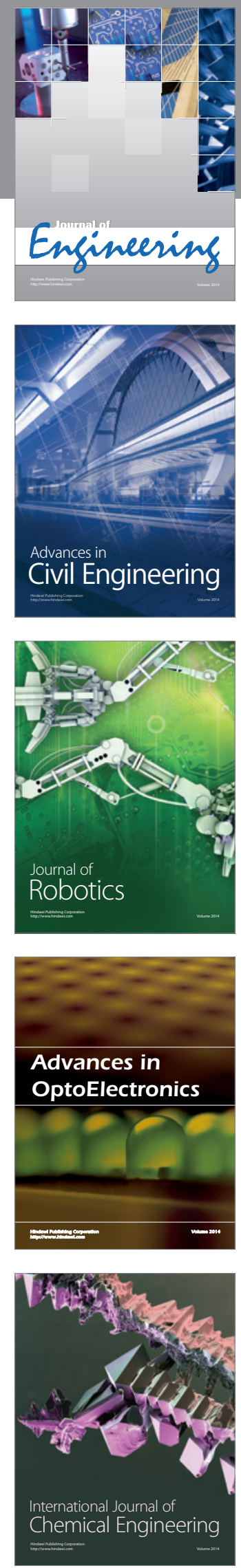

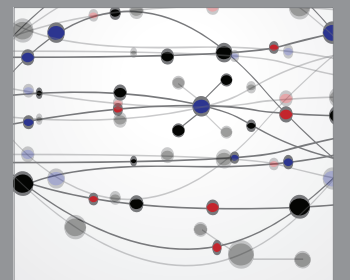

The Scientific World Journal
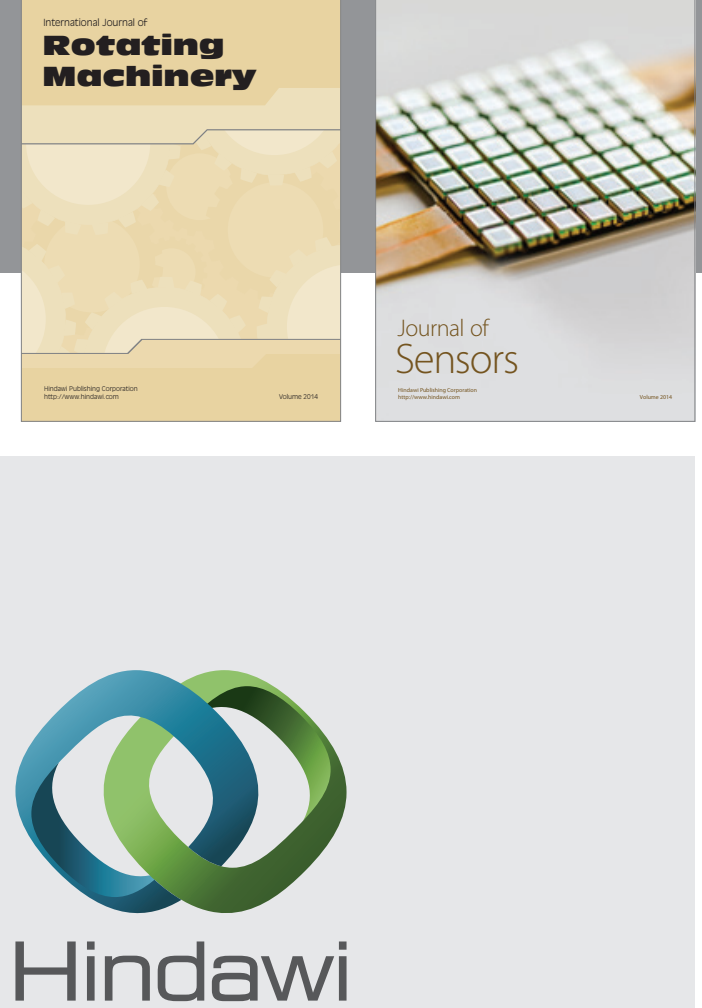

Submit your manuscripts at http://www.hindawi.com
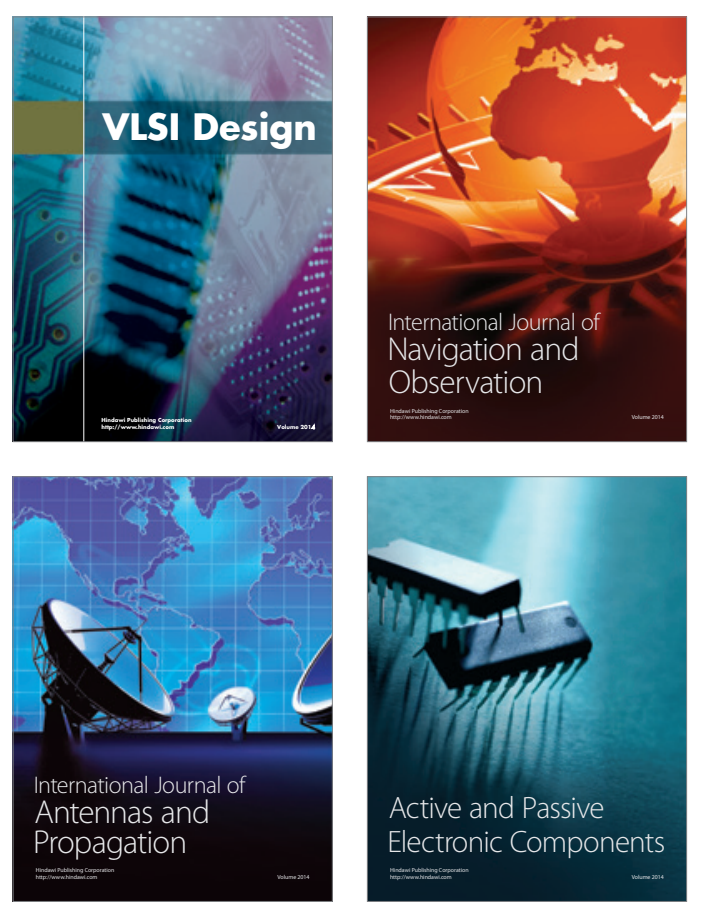
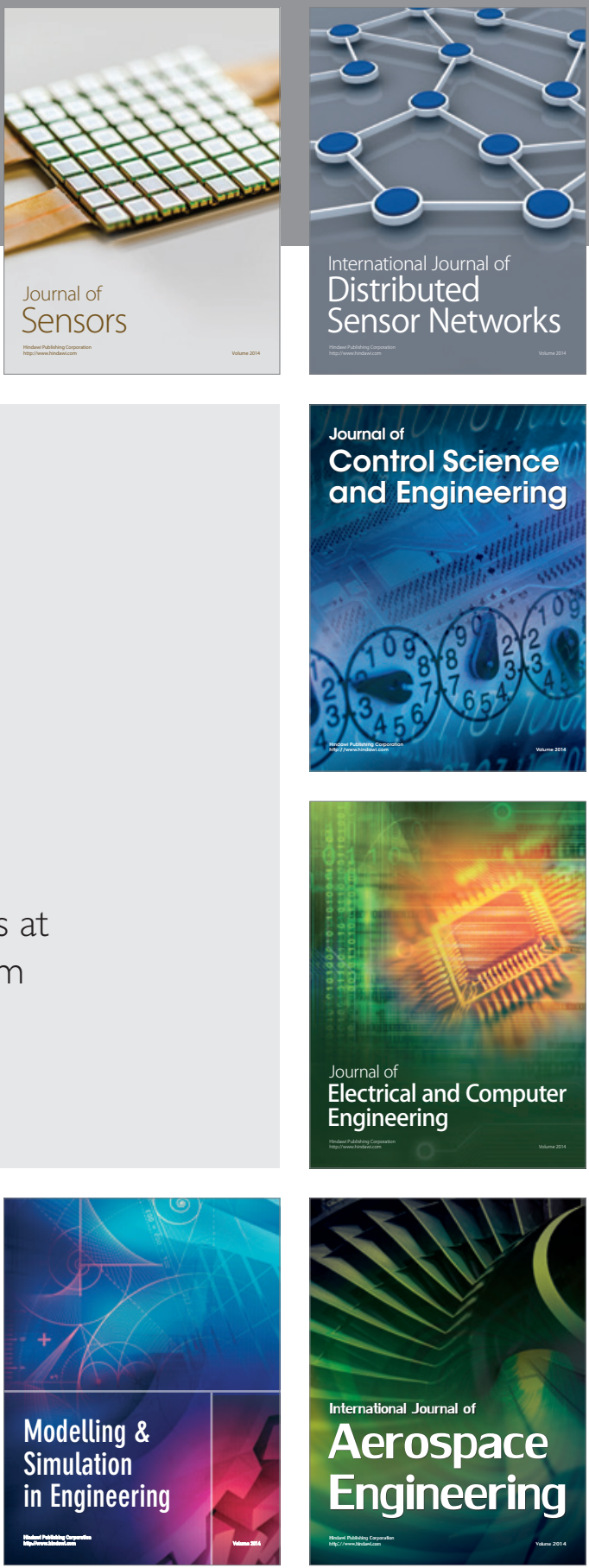

Journal of

Control Science

and Engineering
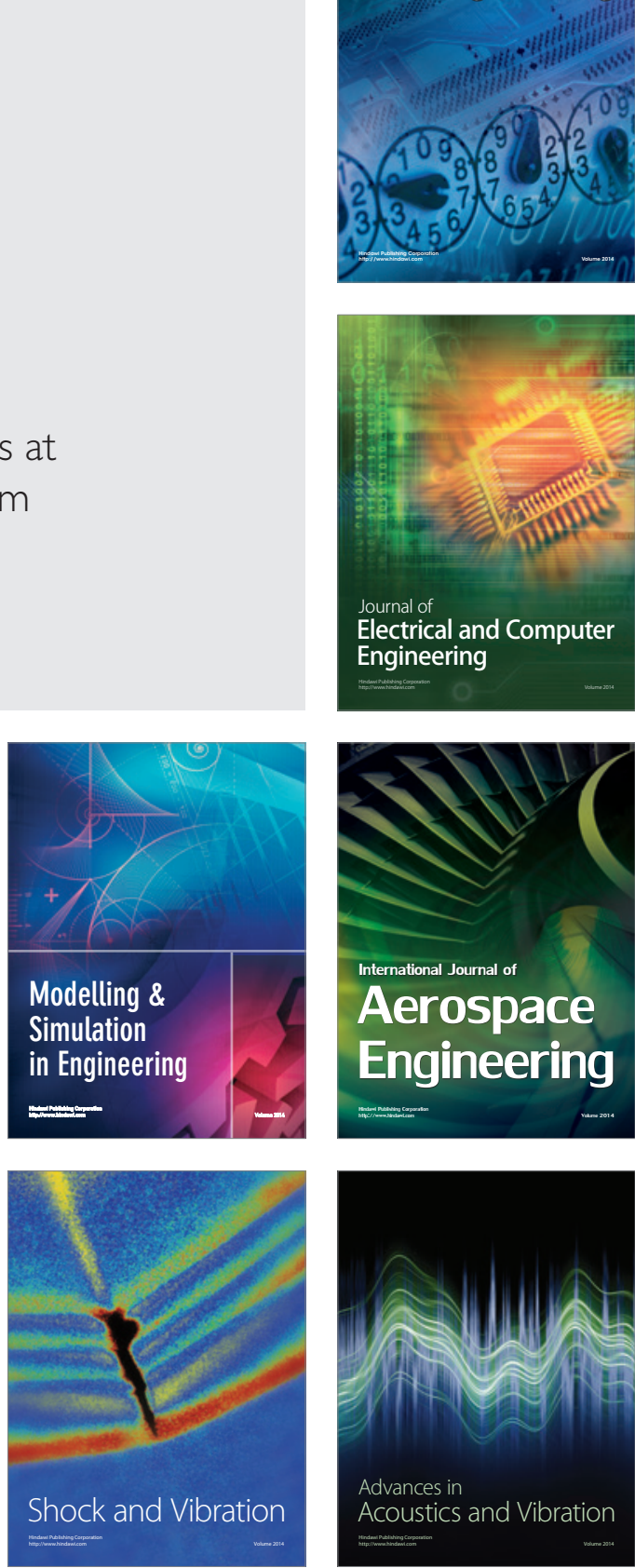